\title{
INFLUENCIA DE LA FORMULACIÓN DEL ENUNCIADO Y DEL CONTROL DIDÁCTICO SOBRE LA ACTIVIDAD INTELECTUAL DE LOS ALUMNOS EN LA RESOLUCIÓN DE PROBLEMAS
}

LANGLOIS, F., GRÉA, J. y VIARD, J.

LIRDIMS. Université Lyon I. 43 Boulevard du 11 Novembre 1918. 69622 Villeurbanne-Cédex.

\section{SUMMARY}

Using as reference the scientist instead of the expert for analysing the pupil's behaviour in probiem solving, we proposed to students (17 years old) closed and open problems. The great diversity of the students' productions and their analysis in the theoretical framework of didactical contract, oblige us to build two analysis grids: one of the students' activities and the other one for the finalities which we attribute to those activities. We show how ranging the strategy of resolution depends on the text of the problem.

\section{INTRODUCCIÓN}

En fúsica, la actividad de resolución de problemas ha dado lugar a una abundante literatura desde los años 80. De su examen resulta que no todos los autores tienen la misma concepción acerca del problema de física.

\section{Diferentes tipos de problemas asociados a diferentes tipos de enunciados}

Entre Ios trabajos propuestos, algunos no ponen en cuestión el concepto corriente de problema asociado a los enunciados estándar que se encuentran en los manuales de enseñanza. Se limitan, en el mejor de los casos, a caracterizar la diferencia de comportamiento entre el experto que sabe resolver estos problemas y el novicio, o entre los «buenos» y los «no tan buenos estudiantes» (Chi et al. 1981, Larkin et al. 1980, Larkin 1983). Del mismo modo que el ámbito de competencia del experto está limitado, los enunciados estándar se refieren siempre a un ámbito preciso y restringido de una enseñanza y a sus soluciones tipo. Además, igual que el experto, el alumno siempre ha tenido previamente conocimiento de un problema considerado como prototipo del que se le plantea.

Estas características llevan a otros autores a decir que los "problemas de física" planteados de esta forma no son verdaderos problemas $y$, en particular, no son problemas que permitan un verdadero avance científico (Gil Pérez et al. 1987). Este análisis ha llevado a los equipos de Dumas-Carré y Gil a adoptar un punto de vista completamente distinto al de los primeros autores citados, proponiendo como modelo de comportamiento, para el alumno, el del investigador, más que el del experto, y resumiendo las principales características del procedimiento del investigador en los términos siguientes:

«- El aspecto ambiguo de los verdaderos problemas; el hecho de que no estén, desde el principio, claramente formulados y modelizados y que falte por hacer la clarificación del objetivo y de la modelización (para inscribirlo en un marco teórico conocido); 
- el aspecto hipotético, incierto, sujeto a la continua puesta en cuestión propia de todo trabajo de investigación;

- como consecuencia de los dos puntos anteriores, el hecho de no encontrase, desde el principio, con los datos a tratar; estos datos hay que construirlos a partir de la modelización realizada y en el marco đe las hipótesis conservadas;

- la necesidad de no aceptar un resultado sin comprobación frente a, por una parte, las hipótesis de salida y, por otra, al corpus de los conocimientos ya adquiridos» (Dumas-Carré et al. 1989).

Este análisis ha llevado a sus autores a proponer un nuevo tipo de enunciado que ya no contiene ningún dato literal ni numérico, con el fin de obligar al alumno a formular hipótesis y a tomar opciones estratégicas. Nosotros, por nuestra parte, hemos propuesto a los alumnos tanto enunciados estándar como enunciados abiertos, proporcionándonos los enunciados estándar una referencia que permite destacar, por comparación, la actividad específica de los alumnos en la resolución de los nuevos tipos de problemas. La supresión de datos en un enunciado y de su corolario, la resolución dirigida por esos datos, viene a ser la ruptura de la convención habitual implícita establecida entre el enseñante y sus alumnos, a la que los profesionales de la didáctica de las matemáticas llaman el contrato didáctico (Brousseau 1986). Ésta es la razón por la cual, a menudo, designaremos en adelante a los enunciados estándar con el término de enunciados de tipo contrato (ent el sentido del contrato didáctico) y a los demás con el término de enunciados sin contrato; entre estos últimos, estableceremos una distinción según su grado de alejamiento del contrato didáctico habitual. La referencia al concepto de contrato didáctico no se limitará, sin embargo, a una categorización de los diferentes tipos de enunciados. En efecto, si en la resolución de problemas, la forma del enunciado es, evidentemente, un elemento determinante del contrato didáctico, el fundamento de este contrato no se limita a ese sólo elemento. Hay, en la práctica đe la clase, muchas otras convenciones, a menudo implícitas, que van a pesar en las decisiones que los alumnos se permiten tomar o no tomar a lo largo de la actividad de ta resolución. Esta observación nos lleva a pensar que no basta con cambiar el enunciado del problema para obtener de los alumnos una práctica de resolución próxima a la del investigador. Ésta es la razón por la que, con el fin de situar a los alumnos lo más cerca posible de la situación del investigador, les hemos propuesto no solamente enunciados muy abiertos sino que además les hemos dado, por una parte, la posibilidad de tener una actividad experimental y, por otra, un tiempo de teflexión superior al tiempo didáctico habitual.

Estas dos últimas características y la interpretación en términos de contrato didáctico de ciertas reacciones de los alumnos han complicado el análisis respecto al de los enunciados papel-y-lápiz, a los cuales se habían restringido los autores precedentes y ha requerido la elaboración đe una herramienta específica de análisis.

\section{La dificultad del análisis y la necesidad de una herramienta específica}

La dificultad de este proyecto reside en el análisis de los protocolos; en efecto, el carácter tan abierto de los enunciados, la duración del trabajo y el abanico de conocimientos de los alumnos les permiten desarrollar numerosas estrategias. Debido a ello, no hemos hecho más que algunos estudios de casos fuera de clase. A continuación, hemos intentado caracterizar el conjunto de las estrategias de los alumnos, a partir de las actividades realizadas y de las finalidades percibidas en esas actividades. Ello nos ha conducido a elaborar dos redes de análisis. Ambas redes son pertinentes para las situaciones de los problemas de física. Sin embargo, la primera red, relativa a las actividades de Ios alumnos es independiente de la disciplina $y$, por tanto, se puede utilizar por todo observador y puede ser precisada, a continuación, a través de las categorías de referencia en física (formalismo, magnitudes, dominio matemático, teórica). No ocurre lo mismo con la segunda red, que ha sido establecida a partir de elementos percibidos en el aspecto disciplinario de la resolución de problemas por los alumnos. Esta red es, a priori, pertinente para examinar las finalidades de todas las tareas de la resolución de problemas de física. Ello nos ha permitido obtener grandes categorías de estrategias de resolución de problemas en relación con la formulación de los enunciados, pero también en relación con los diferentes recursos posibles a la experimentación.

Antes de abordar una exposición más detallada, nos falta decir una palabra acerca de la historia de este trabajo. Tiene por origen un estudio de los procedimientos de verificación de resultados, utilizados por los alumnos en la resolución de problemas, emprendido por uno de nosotros (Khantine-Langlois 1988). A continuación, los protocolos recogidos han sido utilizados en parte para Ilustrar una reflexión sobre la actividad de mođelización en física y ha dado lugar a una publicación (Tiberghien, Langlois 1991). Ningún análisis sistemático de las diferentes estrategias utilizadas por los alumnos en relación con el tipo de contrato didáctico implicado por la forma del enunciado y el contexto de la enseñanza había sido presentado hasta ahora. Este análisis constituye el objetivo de la presente publicación.

En Io que sigue, tras haber detallado las condiciones de nuestra experimentación y de la naturaleza de los problemas planteados, definimos las dos redes de análisis, ilustrándolas con citas extraídas de los protocolos estudiados. Finalmente, indicamos las estrategias puestas de relieve por la utilización de estas redes.

\section{RECOGIDA DE DATOS}

Estos đatos provienen esencialmente de la grabación đe los diálogos de los alumnos durante la resolución de los problemas. 


\section{Condiciones de realización de la grabación}

Todas las grabaciones se han hecho con alumnos voluntarios, fuera de la clase y sin límite de tiempo. Los alumnos estaban informados de nuestras motivaciones y de la ausencia de evaluación de su participación. Sin embargo, todas las grabaciones tuvieron lugar en los locales escolares y con el material experimental de los centros. Cada grabación duró una hora y media aproximadamente.

Siempre se proporcionaba el enunciado por escrito. Los alumnos trabajaron siempre en grupos de dos, con la consigna de elaborar una solución única común. Se grabaron todos los diálogos y se recogieron todos los borradores escritos. Finalmente, cada grabación estuvo seguida por dos observadores que anotaron las acciones de los alumnos que acompañaban a los diálogos y, sobre todo, las ausencias de diálogo. De este modo, observamos a 19 grupos de 2 alumnos cada uno. Esta investigación formaba parte de una investigación más amplia sobre la enseranza de la energía. El contenido pedagógico derivado corresponde, por tanto, al programa de «energía» de los primeros cursos de ciencias de los liceos franceses (programa 1982). Puesto que nuestra investigación no tenía relación directa con el aprendizaje de los conocimientos, sino con su movilización, nos aseguramos, a través de los enseñantes, de que esta parte del programa ya había sido cubierta. Se dio a los estudiantes tres tipos de enunciados diferentes. Más adelante, presentamos el análisis detallado. Los enunciados reseñados por Ia y Ib son familiares a los alumnos, puesto que son de la misma naturaleza que los que se les presenta a lo largo de la escolarización. Los enunciados reseñados por II y III corresponden, por el contrario, a situaciones originales no familiares a los alumnos. El cuadro I resume estas condiciones.

\section{Análisis de los problemas planteados}

Los enunciados del tipo I son enunciados que pueden encontrarse prácticamente en todos los manuales, bajo formas muy semejantes (vienen reproducidos en el anexo).
Estos enunciados inducen a comportamientos escolares que no comportan, a priori, la aportación de hipótesis por parte de los alumnos. Para que aparezcan hipótesis ${ }^{1}$ (conjeturas) que puedan conducir a validaciones en las pesquisas de los alumnos, era necesario proponer, de antemano, otros enunciados distintos a los del tipo I. Dos condicionantes, planteados a partir de los caracteres inhibidores de los problemas de tipo I, han influido sobre su elaboración. El primero consistía en no limitarse simplemente a la determinación experimental de los datos numéricos (cosa que no bubiera producido más que indicaciones de la habilidad experimental de Ios alumnos); teníamos, por tanto, que excluir los dispositivos más habitualmente utilizados en los cursos escolares: plano inclinado, caída libre. La segunda consistía en no introducir un dispositivo demasiado sofisticado, con el fín de que los alumnos pudieran apropiárselo fácilmente; de ahí la idea de considerar un fenómeno conoci. do de todos pero tradicionalmente poco estudiado: el rebote de bolas de materiales diversos. Las bolas facilitadas eran: bolas de juegos infantiles de material plástico que rebotaban más o menos; bolas de acero; y una bola escasamente distinta a las otras al tacto, pero con un comportamiento sorprendente, puesto que no rebotaba en absoluto (en adelante nos referiremos a ella como «bola anormal").

Los otros dos enunciados (problemas II y III) no son en absoluto del tipo contrato y son mucho más abiertos que los anteriores. En efecto, en los dos casos, los alumnos, dejados a su aire, deben analizar un fenómeno, seleccionar las magnitudes pertinentes, definir las medidas a efectuar para conocer esas magnitudes, elegir una técnica experimental, aplicarla y explotar los resultados. Los alumnos tenían a su disposición, ađemás de las bolas, material colocado sin orden en los estantes: regla, baianza, cronómetro, pie de rey, tabla de mađera, calculadora, y la posibilidad de pedir cualquier otro objeto disponible en el laboratorio del instituto del que pudieran tener necesidad.

Si bien los entornos materiales de estas dos situacionesproblema eran semejantes, los dos enunciados, por el contrario, eran muy distintos:

Cuadro I

Condiciones de presentación.

\begin{tabular}{|c|c|c|c|c|}
\hline Problema & $\begin{array}{l}\text { Número de } \\
\text { protocolos }\end{array}$ & $\begin{array}{l}\text { Número de } \\
\text { alumnos }\end{array}$ & Nivel & fecha \\
\hline la y lb & 6 & 12 (3 liceos) & en $1 \mathrm{r}$. curso & $12-87$ a $3-88$ \\
\hline II & 3 & 6 & fin 1t. curso & 7.87 \\
\hline III & 2 & $\begin{array}{l}4 \text { (habiendo ya } \\
\text { trabajado on I) }\end{array}$ & $\begin{array}{c}\text { principio del } \\
\text { curso pre-universitario }\end{array}$ & $11-88$ \\
\hline
\end{tabular}


- El enunciado II contiene términos específicos de física (propiedad, interacción...) y su sintaxis es próxima a la de los problemas "contrato" (se quiere estudiar, para ello se estudia, se interpretará...). Ni la propiedad a estudiar, ni el fenómeno quedan precisados; de ahí la posibilidad de diversas interpretaciones, hipótesis y validaciones. Por contra, la interpretación en términos de energia se refiere a una parte del curso, por tanto, a una modelización muy precisa del fenómeno y se orienta muy claramente a la selección de las magnitudes pertinentes. Además, con este enunciado, los alumnos disponían de todas las bolas desde el principio.

- En el enunciado III, hemos presentado el fenómeno a estudiar sin citar ninguna magnitud. Por tanto, el enunciado no sugiere ninguna modelización. El vocabulario elegido excluye intencionadamente todo término «cientúfico». Por el contrario, hemos Iimitado las posibilidades experimentales, puesto que con la primera pregunta no se facilitaban más que dos bolas del mismo material pero de tamaños distintos. Hasta que los alumnos consideraban que ya habían terminado no se les sometía a la segunda pregunta, en la que se les proponían otras dos bolas de otro material (pero siempre de materiales de comportamiento «normal»).

Para estos dos problemas, es imposible obtener una solución tipo. Evidentemente, no es de esperar un estudio muy completo del contacto bola - suelo. El análisis a priori de esta situación no preveía más que la posibilidad de que Ios alumnos llegaran, en eI mejor de los casos, a la noción de «coeficiente de restitución", tal como se da en los cursos de física general clásica, por ejemplo, Bruhat ${ }^{2}$. (Esta descripción puramente cinemática no tiene en cuenta los efectos térmicos.)

Los escenarios esperados por los investigadores eran los siguientes:

- Con el enunciado II, los alumnos considerarían, tras algunos ensayos de caída de las diferentes bolas, si la altura del rebote dependía o no dependía de la nafuraleza del material, de la masa, del volumen, introducirían la interpretación energética del fenómeno y darían las explicaciones del proceso de contacto. No habíamos previsto el hecho de que los alumnos pudieran pensar en ningún otro fenómeno que el rebote como mecanismo de interpretación; sin embargo, uno de los grupos consideró los rozamientos.

- Con el enunciado III, nosotros esperábamos un estudio más completo del rebote de la primera bola: medida de la altura del primer rebote en función de la altura de partida, trazado de la curva, interpretación en términos de energía como en el otro caso, estudio en función del material sobre el que se realiza el rebote (ésa es la razón por la que habíamos facilitado una tabla de madera, siendo el suelo de baldosa), etc. Pensábamos enmarcar mejor el mecanismo de validación, ya que, según las hipótesis hechas y las magnitudes introducidas, el mismo trabajo podía entonces repetirse o no con la segunda bola. Las predicciones para el segundo juego de bolas obligaría, pensábamos, a los alumnos a precisar sus nuevas hipótesis y nos permitiría saber cuáles serían validadas. Los ensayos posteriores con otras bolas debian permitir la verificación.

En ambos casos, habíamos admitido explícitamente que los alumnos abordarían el problema en términos dic mecánica (lo que correspondía a la parte del programa de referencia), pero no ocurrió siempre así.

En adelante, las citas de los alumnos están escritas en cursiva, excepto las citas que corresponden a la relectura de los enunciados. Los nombres van seguidos, entre paréntesis, por el número del problema tratado.

\section{Las redes de análisis}

La primera red se refiere a las actividades de los alumnos.

\section{Red de actividades}

Hemos clasificado esas actividades por orden creciente de abstracción. Están asociadas a actitudes e intenciones consideradas como elementos de estrategia de resolución de la tarea según el decir del alumno o de la evidencia cotidiana:

A1. Actividad de naturaleza práctica: Observaciones, pruebas, medidas (referidas a la experimentación o a la práctica cotidiana). Se trata de actividades realmente prácticas que conciernen bien a las percepciones o a las actitudes (sentidos), o bien a las medidas (aparatos), y que proporcionan datos cualitativos o numéricos.

A2. Actividad de naturaleza simbólica: Aplicación de un procedimiento de tratamiento matemático de las informaciones, trazado de una curva, linealización (respecto a la formalización matemática). Durante esta actividad, el alumno establece nuevas relaciones matemáticas sin ocuparse de su interpretación. Esta actividad se hace a partir de los resultados de la actividad A1 o de los datos del enunciado.

A3. Actividad de naturaleza teórica: Aplicación de un procedimiento de tratamiento de las informaciones en el marco de un modelo identificable ${ }^{3}$ : enunciado de principios, de leyes, elección de magnitudes y de interacciones (respecto a una teoría física). Durante esta actividad, el alumno selecciona magnitudes y relaciones entre magnitudes, manifestando que son pertinentes para la resolución del problema.

A4. Actividades de naturaleza especulativa: Apelación a conjeturas, es decir, enunciado o formulación de hipótesis (respecto a la construcción de un modelo por parte del alumno). Al acabar esta actividad, el alumno selecciona magnitudes sin saber si su elección es pertinente para la resolución. 


\section{Red de finalidades}

La segunda red trata de las finalidades que nosotros atribuimos, en el marco de la teoría didáctica de la resolución de problemas de física, a las actividades clasificadas en la primera red. Estas finalidades pueden ser de cuatro clases y las hemos clasificado en el orden de aparición más frecuente durante la resolución del problema por los alumnos:

F1. La comprensión (del enunciado del problema, de los fenómenos) en términos đe explicitaciones y de aplicación de procedimientos, de actividades experimentaleso de predicciones.

F2. La validación de una conjetura (formal o como consecuencia de la elección de un mođelo), de uso personal o social, en términos de justificación.

F3. La elaboración de un procedimiento, para la obtención de un resultado, en términos de cálculos o de organización. Este procedimiento puede ser de carácter empírico, matemático o teórico (físico).

F4. La verificación de un resultado (parcial o final) en términos de comparaciones con datos que se derivan de conocimientos en otros domintos.

Precisamos ahora el sentido que atribuimos a estas finalidades.

La comprensión ${ }^{4}$. Distinguimos la comprensión del enunciado de la comprensión del fenómeno. Una no implica necesariamente la otra. En efecto, los problemas que el alumno tiene que resolver en el marco escolar siempre le vienen propuestos por el enseñante. Se trata siempre de una demanda que le es externa. En este contexto, diremos que hay una comprensión del enunciado cuando la respuesta a suministrar venga explicitada por el alumno (ejemplo: la respuesta sería el valor numérico de la magnitud A, o la respuesta sería el trazado de una curva que represente la magnitud $\mathrm{A}$, en función de la magnitud $B$, etc.). Esta comprensión puede no ser más que parcial y revisada durante la resolución. Existe comprensión del enunciado cuando los fines y las actividades de los alumnos convergen con los que espera el enseñante que ha propuesto el problema. La comprensión, incluso parcial, del enunciado desencadena la aplicación de un procedimiento. Veamos un ejemplo de parte del diálogo en el que reconocemos una etapa de comprensión del enunciado:

Corinne (Ia): De entrada, la primera pregunta: ¿Cuál es la fuerza que se opone al desplazamiento? Por tanto, para la primera pregunta hay que utilizar una gráfica.

Lo mismo para la comprensión del fenómeno:

Jean-François (III): No, no se puede; depende del calor que absorba. Si rebota menos, absorbe, no... pierde energía cinética; por tanto, se calienta, gana energía calorifica.
La comprensión del fenómeno se traduce a menudo en términos de esquematización y de modelización. No se trata solamente de responder a lo que el profesor espera sino de la necesidad personal de apropiación. Ello implica la formulación de conjeturas y la necesidad de validarlas mediante la experimentación.

La validación. Utilizamos este término a partir de las producciones de los alumnos que se derivan de la decisión de conservar o rechazar una conjetura, sea esta última explícita o no. Las conjeturas de los alumnos pueden ser de naturaleza variada: elección de la depenđencia del fenómero respecto a una variable, o, en el caso de que ésta ya haya sido validada, elección de una Iey matemática que traduzca esa dependencia. Diremos que existe valìdación cada vez que, tras ciertas experimentaciones, medidas o cálculos, los alumnos conserven la conjetura y la consideren como un dato para lo que resta del trabajo. Si, por el contrario, se rechaza la conjetura y se reemplaza por otra, no existe validación. Las conjeturas se esbozan a partir de los enunciados o bien son implícitas y planteadas por el investigador, pero, contrariamente a la verificación, la validación no se explicita jamás: el alumno no dice nunca «yo valido» del mismo modo que diría "yo verifico». Por ejemplo, Florent y Laurence (II) afirman que «cuanto menor es la variación de la energía potencial, más elástica es» y que «cuanto más elástica es la sustancia, más rebota». Por tanto, hacen la conjetura de que la bola de materia plástica es más elástica que la bola đe acero. La experiencia les sorprende: "No está tan claro como parece, esto (la bola de acero) rebota más que esto (la bola de plástico)." Sin embargo, cuando, tras sus medidas y sus cálculos de energía potencial, constatan que la variación đe energía es más importante para el plástico, lo explican por una diferencia de masas, cosa que no cuestiona la conjetura acerca de la sustancia: esta conjetura no queda, por tanto, invalidada.

Laurence (II): Ah, entonces es por eso por lo que se puede decir que el acero es menos elástico que el plástico.

Florent (II): Solamente en el caso de la experiencia; como las masas son claramente diferentes, vemos que el acero rebota más alto.

La elaboración de un procedimiento. Según J.M. Hoc (1987), un procedimiento es un «sistema previamente adquirido de operaciones coordinadas con el fin de obtener un cierto objetivon. Puede tratarse de un procedimiento de carácter práctico (compuesto de medidas), 0 matemático (linealización), o teórico (aplicación del teorema de la energía cinética). La elaboración de un procedimiento consiste, por tanto, en imaginar un protocolo de operaciones sucesivas realizables para acabar en un resultado preciso. En la red de actividades, cuando hablamos de la aplicación de un procedimiento, se trata de un procedimiento ya elaborado y que termina siempre en la obtención de un resultado.

Jean-François (III): Lo que haría falta es arrancar de diferentes alturas. Después se mide cada vez la altura (del rebote); haremos una gráfica. 
Trazar una gráfica es un procedimiento conocido por los dos alumnos, que no requiere comentarios.

La verificación. Incluimos en este epígrafe toda revisión de un resultado cualitativo o cuantitativo alcanzado como producto de un procedimiento, pero que, debido al hecho de la utilización de un razonamiento o de un cálculo más o menos largo, comporta para el alumno un riesgo de error más o menos fortuito. Existe verificación de un resultado cuando ya se tiene de él una cierta idea. La no conformidad de este resultado con la idea que se tenía de él no pone en cuestión, de entrada, Ia opinión del alumno, y solamente provoca el hecho de plantearse el control de los métodos técnicos utilizados para obtener esta solución. En este contexto, solamente los alumnos que obtienen resultados tienen, efectivamente, la posibiIidad de verificarlos. La actividad de verificación puede ser voluntaria o explícita; el alumno dice claramente: «hago esto para verificar».

Laurence (II): [...] tras un cálculo de la energía potencias. Pero me pregunto si teóricamente se tenía que hallar un valor negativo.

Corinne (Ia) (Tras haber calculado un trabajo utilizando la relación $\mathrm{W}=\mathrm{F} \times \mathrm{L})$ : Estaba mirando si, haciendo también la suma de los trabajos igual a cero, se obtenía el mismo resultado.

\section{RESULTADO DEL ANÁLISIS}

Antes de pasar revista a los diferentes tipos de problemas, presentamos dos observaciones que se aplican al conjunto de los enunciados.

\section{Observaciones generales}

Una primera observación hecha a partir de los comentarios de los alumnos que han hecho dos tipos de problemas concierne al estado de ánimo en el cual abordan estos problemas. En el caso de los problemas de tipo I, están convencidos de contar con todos los elementos para responder a las preguntas. Si no lo consiguen es, creen ellos, por falta de trabajo o porque "no son buenos». Por el contrario, para el resto de los enunciados, los alumnos dudan de su capacidad para resolver el problema, por falta de conocimientos. Este fenómeno nos parece revelador de la presencia del contrato didáctico en los alumnos.

Laurent (II): El concepto al que yo llamo rigidez es, quizá, un poco demasiado complicado, a mi entender... pero es que tenemos los medios para resolver el problema... pero no podemos, no tenemos ni idea de la composición... no se ha hablado de...

Ludovic (III): Bueno, podemos decir que no se puede encontrar una ley sobre esto, porque es, en todo caso... a nuestro nivel.
Jean-François (III): Me he planteado que esto debe ser esto, pero no se puede hacer.

La segunđa observación se refiere al gran carácter abierto de los problemas de tipos II y III. Ent efecto, nos hemos visto llevados a distinguir entre "una resolución directa» y «una resolución indirecta». La resolución directa corresponde a una resolución lineal, es decir, sin actividad abandonada, mientras que la resolución indirecta implica vueltas atrás y abandonos. A veces, ciertos alumnos anuncian las resoluciones directas diciendo lo que les gustaría hacer, pero que no lo consiguen.

Daremos, en todo caso, el procedimiento de resolución que nos permitirá el análisis de la estrategia de los alumnos en términos de comprensión, de verificación y de validación.

\section{Problemas estándar (Ia y Ib) 4.2.1}

La resolución directa de un problema estándar, tal como la contempla el enseñante y tal como los alumnos Ia aplican, puede resumirse mediante el cuadro II.

Cuadro III

Desarrollo directo del problema tipo II.

\begin{tabular}{|c|c|c|c|c|}
\hline \multicolumn{2}{|c|}{ Actividad } & \multicolumn{2}{|l|}{ Finalidad } & Resultado \\
\hline $\begin{array}{l}\text { Lectura, } \\
\text { tratamiento } \\
\text { físico }\end{array}$ & A3 & $\begin{array}{l}\text { Comprensión del } \\
\text { enunciado }\end{array}$ & F1 & $\begin{array}{l}\text { Selección de } \\
\text { formulas }\end{array}$ \\
\hline Experiencia & $\mathrm{Al} \mathrm{A} 2$ & Procedimiento & F3 & $\begin{array}{l}1 \text { ó } 2 \text { resultados } \\
\text { numéricos }\end{array}$ \\
\hline $\begin{array}{l}\text { Tratamiento } \\
\text { f́sico }\end{array}$ & $\mathrm{A} 3 \mathrm{~A} 4$ & $\begin{array}{l}\text { Verificación } \\
\text { Validación }\end{array}$ & $\begin{array}{l}\text { F4 } \\
\text { F2 }\end{array}$ & Conjetura \\
\hline
\end{tabular}

Debido a las consignas del contrato, no puede aparecer aquí ninguna actividad práctica $\mathrm{A} 1$. Se constata, por otro lado, la ausencia de actividad A2. Puesto que las actividades de los alumnos son esencialmente del tipo A3, es decir, siempre referiđas a la modelización y a la finalidad F3, en ningún momento aparece la conjetura. Este esquema se reproduce, tal cual, en cada pregunta del enunciado. El «tratamiento físico» de la información consiste esencialmente en el enunciado de un principio o de una relación entre las magnitudes citadas por el enunciado o por el problema prototipo reconocido y relevante de la física enseñada.

Jean-François (Ia): V es constante, la suma de las fuerzas es igual a 0 y la suma de los trabajos es igual a 0.

Odile (Ia): El trabajo es una función de ly tenemos 1 en función del tiempo.

Encontramos aquí un comportamiento ya abundantemente descrito (por ejemplo, Dumas-Carré 1987). El 
tratamiento consiste, a continuación, en aplicar un procedimiento enseñado (identificable por idéntico al de la solución tipo), constituido por diversos elementos (dibujo, ecuaciones, cálculos numéricos...). Según los datos numéricos y la pregunta planteada, el alumno procede a transformaciones matemáticas con sus ecuaciones para aislar las cantidades pedidas.

La comprensión del enunciado viene asociada a la identificación de un procedimiento prototipo. Un buen prototipo se manifiesta por una buena adecuación entre el conjunto de las magnitudes de las que se dispone y las que están presentes en el prototipo. Una vez reconocido este prototipo, no resulta sorprendente que todos los grupos aborden ia resolución de la misma manera, puesto que el método a aplicar para resolver un problema de dinámica se encuentra en todos los manuales, bajo una u otra forma. Desde el momento en que el alumno identifica inmediatamente el prototipo, ya no estamos realmente en posición de decir sobre la base de qué criterios se realiza esta identificación. Puede tratarse, quizá, de simples criterios superficiales tales como la semejanza de las variables con aquéllas de una situación prototipo ya estudiada o, tal vez, un análisis físico muy rápido del enunciado, como parece indicar el tratamiento por parte de los alumnos de los enunciados de los problemas no estándar (ver más adelante) y simultáneamente la utílización de criterios de verificación relacionados con el contrato (Khantine-Langlois 1988). Es evidente que la memoria desempeña aquí un papel importante:

Aline (Ib): Cuando tenemos un ejercicio como éste, repaso un ejercicio ya hecho para ver cómo lo he hecho. No sólo lo busco, investigo lo que he hecho antes e intento hacerlo parecido; cuando no lo consigo es que no me acuerdo.

Nos hemos encontrado con etapas de verificación en este tipo de solución, pero no es algo sistemático. Estas verificaciones son esencialmente del tipo contrato, bien porque son idénticas a las realizadas en las soluciones prototipo, bien porque siguen las prescripciones que se derivan de indicaciones explícitas del profesor y no đe elementos pertinentes en física.

\section{Análisis de las estrategias de resolución}

Hemos observado un desarrollo lineal en tres grupos de seis. En los otros tres grupos, la resolución ha sido indirecta, puesto que la identificación del prototipo no ha sido inmediata. La comprensión del enunciado no está en cuestión, en el sentido de que todos los alumnos han tenido una idea precisa de lo que pensaban que tenian que hacer: aplicar un procedimiento conocido, recuperar algo ya hecho. Así, Aline y Christophe (Ib) prueban sucesivamente el esquema de la "caída libre», después el del «péndulo físico» y después el «teorema de la energía cinética».

Christophe (Ib): Vamos a utilizar la energia potencial; espera que me acuerde de todo.
Aline (Ib): Es que no podemos decir que sea un cuerpo en caída libre y tú sabes que hemos aprendido a calcular la velocidad de un cuerpo en caída libre.

Christophe (Ib): Sí, pero esto es el péndulo físico. No sé si te acuerdas que hemos hecho el péndulo físico.

En estas condiciones, la validación, tal como la hemos defínido, no puede aparecer como resultado de la resoitición directa de un problema estándar. En efecto, la modelización y las hipótesis necesarias para esta modelización ya han sido hechas y validadas en eI curso. Que esta validación le haya convencido o no al alumno no le lleva a sentir la necesidad de volver a ella. El alumno tiene siempre a su disposición un procedimiento de resolución y es a partir de la aplicación de este procedimiento cuando, eventualmente, puede verse impelido a volver a ponerlo en cuestión. Esta situación no resulta siempre fácil de descubrir en clase puesto que, en ese momento, el alumno o bien puede «no hacer nada» o bien puede aplicar ebrutalmente» este procedimiento para obtener «la buena solución» ( $y$ una buena nota) sin estar de acuerdo con el mismo. Nos hemos encontrado con este caso en uno de los grupos: Abed y Nora (Ia) han observado que el automóvil circulaba a una velocidad constante. Ellos conocían el principio de la intercia. En el momento de aplicarla a este caso concreto, han dudado porque, en el fondo, la validez de este principio no les parecía garantizada. En un primer momento, Abed renuncia a utilizarlo. En un segundo momento, Nora se decide a aplicar el procedimiento, a falta de otra cosa mejor:

Nora (Ib): Diciendo que la suma de las fuerzas es igual a $0, y \ldots$ ¿por qué quieres que sea igual a 0?... no sé, cuando haces que v sea igual a constante, cuando la velocidad es constante, no puedes tener que la suma de las fuerzas sea igual a 0. Bueno, probemos, pero no es seguro que funcione.

A lo largo de la discusión, Abed (lb) precisará:

Abed (Ib): Yo no estoy de acuerdo porque, concretamente, estas dos fuerzas, son las mismas si, pongamos... al extremo de una cuerda, cada una de $100 \mathrm{~N}$ y la cuerda no se va a mover... por tanto, no es posible; el automóvil no puede avanzar a $54 \mathrm{~km} / \mathrm{h}$ con una fuerza de rozamiento y una fuerza motriz iguales.

\section{Problemas no estándar}

Los alumnos han admitido espontáneamente que deben hacer un estudio experimental (actividad AI) aunque el enunciado no lo inđique. La presencia del material y eI contexto bastan. Tal como estaba previsto en el análisis a priori, los dos enunciados han conducido a estrategias muy diferentes. Nos referimos a ellas como «ejercicio» y «trabajos prácticos» concernientes a las actividades escolares habituales de los alumnos, en física. La referencia «ejercicio» (problema II) Ileva a abordar el enunciado y el material como los problemas de tipo I, en el espíritu del contrato didáctico: «Los enunciados contie- 
nen las informaciones que hay que traducir. Si nos dan tal objeto, es que hay que utilizarlo". Consciente o no, esta actitud es muy frecuente y a los alumnos, incluso a los prevenidos, les cuesta mucho deshacerse de ella. Hemos utilizađo el tếrmino «trabajos prácticos» para el problema III, ya que Ludovic utilizó esas palabras y Ia estrategia desarrollada es, efectivamente, la que se hace seguir a los alumnos en un gran número de sesiones de trabajos prácticos destinadas a introducir nuevas magnitudes (es decir, de hecho, a modelizar). Sin saber a qué aspectos del enunciado se deben estas diferencias, podemos afirmar que la formulación del enunciado contribuye de forma determinante, puesto que el método «directo" ha sido el mismo para los tres grupos del problema II y ha estado ausente de los dos grupos del problema III. Hablamos de método directo en la medida en que los alumnos han enunciado una estrategia, que se han mantenido en ella y que estamos en posición de ver los elementos que han perturbado su buen desarrollo.

El cuadro III resume el desarrollo directo del problema de tipo II.

\section{Cuadro III}

Desartrollo directo del problema tipo II.

\begin{tabular}{|c|c|c|c|c|}
\hline \multicolumn{2}{|c|}{ Actividad } & \multicolumn{2}{|c|}{ Finalidad } & Resuitado \\
\hline $\begin{array}{l}\text { Lectura, } \\
\text { tratamiento } \\
\text { fisico }\end{array}$ & A3 & $\begin{array}{l}\text { Comprensión đel } \\
\text { enunciado }\end{array}$ & F1 & $\begin{array}{l}\text { Selección de } \\
\text { fórmułas }\end{array}$ \\
\hline Experiencia & A1 $\mathrm{A} 2$ & Procedimiento & F3 & $\begin{array}{l}1 \text { 6 } 2 \text { resultados } \\
\text { numéricos }\end{array}$ \\
\hline $\begin{array}{l}\text { Tratamiento } \\
\text { físico }\end{array}$ & A3 A4 & $\begin{array}{l}\text { Verificación } \\
\text { Validación }\end{array}$ & $\begin{array}{l}\text { F4 } \\
\text { F2 }\end{array}$ & Conjetura \\
\hline
\end{tabular}

Para los tres grupos, la estrategia de la resolución del problema de tipo II ha sido la misma. Cada elemento del enunciado se asocia a fenómenos o a magnitudes de forma intuitiva, sin justificación, por asociación de ideas.

\section{Laurence(II): La interacción con el suelo es la fuerza del} peso.

Florent: La interacción con el suelo; puedes tener también los rozamientos, las reacciones.

Anne (II): La interacción con el suelo, bueno, es cuando las bolas rebotan. Se interpreta el fenómeno en términos de energía. ¿Qué se puede pensar? Energía potencial, energía cinética. La distinción entre las sustancias es el principio que no comprendo bien.

Ciertas ideas son rechazadas porque resultan imposibles de manejar o aparecen como no pertinentes en el marco de la modelización adquirida. Así:

Florent (II): Tienes propiedades químicas, tienes propiedades mecánicas, químicas; de todas maneras, no se puede hacer.
Laurence (II): La energía; tenemos $E=m c^{2}$.

Florent (II): Solamente aquí hay contactos de energia pero la masa no cambia en cualquier caso.

Las numerosas explicaciones en esta parte del trabajo ponen en relación diferentes elementos adquiridos:

Anne (II): Ésta rebota más alto porque es más ligera y no son las mismas sustancias.

Después de varias idas y venidas, enunciado / explicaciones, se llega a una relación hipotética que toma en consideración todos los elementos adquiridos y manejables en el marco de la modelización sugerida por el enunciado (esencialmente masa y tamaño de las bolas, y energía potencial) y que permite definir la experiencias a realizar.

Anne (II): Si, es eso, cuanto más rebote la bola, menos rozamiento habrá habido con el suelo, por tanto, menor pérdida de energía. Eso es lo que pasa y sabemos que, si la bola que rebota llega a su punto inicial, es decir, al punto desde donde se la ha lanzado, se podrá decir que no habrá habido rozamiento.

Philippe (II): Eso es; bueno, hagamos la experiencia... y también hay una relación con las sustancias y los tamanos diferentes.

La experiencia no es, en este caso, a priori, más que un simple procedimiento destinado a proporcionar los datos numéricos necesarios para seguir el trabajo. Ésa es la razón por la que los alumnos no hacen más una o dos pruebas; su opinión ya está establecida.

La comprensión es, al inicio del trabajo, mucho más una comprensión del enunciado y de la demanda del profesor que una comprensión de un fenómeno físico, tal como muestra el grupo Florent-Laurence (II). En efecto, este grupo se ha lanzado, primero, a estudiar el rozamiento de la bola con el suelo. Bajo la petición del enseñante presente, a continuación ha retomado el trabajo sobre el rebote, manteniendo en la conversación la energía, por imposición del enunciados.

Un efecto imprevisto de la elección del dispositivo material se ha manifestado en las bolas utilizadas por los alumnos para la experiencia. El azar ha introducido diferencias de comportamiento entre los grupos. Florent y Laurence (II) no han utilizado más que un juego de bolas del mismo material y de tamaños, por tanto, de masas, diferentes. Así, han obtenido valores numéricos coherentes con su análisis y se han mantenido en ellos. Han tenido la impresión de dominar completamente el problema y no se han planteado ninguna cuestión sobre el fenómeno. Esta sifuación ha validado completamente sus ideas.

El segundo grupo, Anne y Philippe (II), ha empezado exactamente igual que Florent y Laurence (II). Pero ha querido verificar, a continuación, los resultados obtenidos con las dos bolas de plástico, sustituyéndolas por dos 
bolas de igual masa, pero una de acero y otra de plástico. La no-conformidad de los resultados con lo que ellos esperaban les ha obligado a volver a poner en cuestión las conjeturas que habían hecho implícitamente y a validarlas. Así, se han visto llevados a contemplar el enunciado dentro de un marco más amplio, para estudiar el fenómeno. Además, en el momento en que, finalmente, han utilizado la bola de comportamiento anormal, no se han sentido alterados. Sus reflexiones les habían conducido a numerosas conjeturas y esta nueva situación se ha integrado sin dificultad en sus esquemas de razonamiento.

El tercer grupo, por el contrario, ha dado la impresión de no comprender el enunciado. En efecto, estos dos alumnos (Hêlène y Laurent (II)) han hecho, por puro azar, la primera prueba con la bola anormal. Ello les ha obligado, desde el principio, a tener en cuenta, simultáneamente, la masa y la naturaleza de la sustancia, como dos parámetros esenciales. Además, esta prueba les ha sugerido el caso de choques elásticos o blandos. Debido a este hecho, han movilizado simultáneamente sus conocimientos acerca de dos categorías de fenómenos: la caída y los choques. El conjunto se ha mostrado imposible de manejar; el tratamiento del choque no es posible a falta de magnitudes medibles.

Hélène (II): Pasa algo a nivel del suelo, por tanto, habrá que aislarlo y expresarlo en función de... Bueno, yo quisiera, pero como en estas fórmulas no hay ningún factor sobre lo que es el cuerpo, o sea, de su composición, pues, de hecho, escribir que esto no nos sirve de gran cosa, porque hay que poner algo en forma de ecuación, habría que hacerla intervenir [lo que ellos han llamado rigidez] en una ecuación, en alguna cosa.

Observemos que, con este enunciado, estos dos alumnos no han conternplado, en ningún momento, la posibilidad de introducir lo que han llamado «rigidez» como una nueva magnitud. Está implícito, para ellos, que la solución no puede hacer intervenir más que las magnitudes definidas previamente en el curso. Este becho es, de nuevo, una manifestación muy clara del contrato al que los alumnos están sometidos.

El cuadro IV resume las estrategias de resolución para los tres grupos que han trabajado sobre este problema.

El cuadro $\mathrm{V}$ resume el desarrollo directo del problema III. Dos grupos han trabajado con este enunciado. Para uno, el desarrollo se ha hecho de forma directa (Ludovic y Corinne), al contrario que el otro (Cécile y JeanFrançois). Sin embargo, estos dos últimos alumnos han dicho explícitamente que deseaban proceder de la forma que hemos llamado directa. En esta aproximación muy matemática, el paradigma subyacente es la existencia de invariantes de las que se podrán deducir leyes que se impondrán por sí mismas. Contrariamente a la situación anterior, en este caso está permitido introducir una nueva magnitud, pero esta magnitud debe depender de las magnitudes conocidas. Ludovic lo explica muy claramente en respuesta a la pregunta "¿Por qué tomáis estas medidas?»:
Ludovic (III): Para obtener una recta que nos permita conocer la altura del rebote en función de la altura de caída. Esto quizá nos sirva porque normalmente la altura de rebote debe ser proporcional a la altura de caída... vamos a ver si vemos lo mismo con la otra bola. Si es dos veces menos pesada, si vemos eso, vamos a encontrar dos veces más una cosa como ésta; esto puede ser interesante y así se puede decir que el rebote es función de la masa.

Cuadro IV

Estrategias de resolución del problema II.

\begin{tabular}{|l|l|l|}
\hline \multicolumn{1}{|c|}{ Grupo } & Comprensión de1 enunciado & Validación \\
\hline Laurence Florent & Sí & Sí \\
\hline Anne Philippe & $\begin{array}{l}\text { Sí (tras el fenómeno por } \\
\text { ctapas sucesivas) }\end{array}$ & $\begin{array}{l}\text { Parcial en } \\
\text { cada etapa }\end{array}$ \\
\hline Hélène Laurent & $\begin{array}{l}\text { No (por experiencia } \\
\text { desconcertante) }\end{array}$ & No \\
\hline
\end{tabular}

Cuadro V

Desarrollo directo deI problema III.

\begin{tabular}{|c|c|c|c|c|}
\hline \multicolumn{2}{|c|}{ Actividad } & \multicolumn{2}{|c|}{ Finalidad } & Resultado \\
\hline Lectura & $\begin{array}{l}\text { A3 } \\
\text { A4 }\end{array}$ & Comprensión & F1 & Conjetura \\
\hline Experiencia & A1 & $\begin{array}{l}\text { Procedimiento } \\
\text { (verificacion) }\end{array}$ & F3 F4 & $\begin{array}{l}\text { Conjunto de } \\
\text { resultados } \\
\text { numéricos }\end{array}$ \\
\hline Tratamiento & A2 & $\begin{array}{l}\text { Procedimiento } \\
\text { matemático } \\
\text { (verificación) }\end{array}$ & F3 F4 & Curva \\
\hline $\begin{array}{l}\text { Tratamiento } \\
\text { físico }\end{array}$ & $\begin{array}{l}\text { A3 } \\
\text { A4 }\end{array}$ & Validación & $F_{2}$ & $\begin{array}{l}\text { Nueva } \\
\text { conjetura }\end{array}$ \\
\hline
\end{tabular}

Más adelante:

Ludovic (III): $Y$ ahora, este coeficiente director, habrá que encontrar en función de qué está; por ejemplo, es la masa la que divide el volumen o algo parecido.

Jean-François y Cécile tenían en la cabeza un proyecto idéntico:

Jean-François (III): Lo que haría falta es partir de diferentes alturas, después se mide cada vez la altura [del rebote]; haremos un gráfico y después obtendremos una recta o algo así.

Se puede observar que este método es exactamente el que se propone en los programas para introducir ciertas magnitudes tales como la resistencia eléctrica, la rigidez de un resorte o el índice de un medio y que, en todos los 
casos, se define la nueva magnitud como un coeficiente de propotcionalidad; no resulta, por tanto, sorprendente volver a encontrar esta modalidad de razonamiento en los alumnos.

Es interesante analizar por qué uno de los grupos ha validado su modelo de proporcionalidad y el otro no. Ludovic y Corinne han realizado medidas, han trazado curvas y han calculado los coeficientes directores, a los que ellos llaman coeficientes de rebote, sin buscar una explicación al fenómeno. Además, sus medidas sirven para determinar el coeficiente director de la recta y no la naturaleza de la curva, lo cual explica que tres toscas medidas les hayan bastado. Aunque derivado de la misma estrategia, el trabajo de Cécile y Jean-François no ba culminado, puesto que ha sido perturbado por teorías incompatibles con su protocolo. Cécile ha tratado siempre de utilizar las ecuaciones del movimiento de la bola en caída libre y de remitirse a cálculos hechos en el curso. Jean-François tiene una explicación termodinámica del fenómeno que no cuadra en absoluto con su estrategia experimental. Ello hace que ni uno ni otro hayan podido actuar, tal como muestra este diálogo:

Cécile (III): Podríamos tratar de expresar esta altura [de caída] y esta altura [de rebote] con ecuaciones del movimiento, en función de to algo así; puede que $\mathrm{t}$ se simplifique y que obtengamos valores.

Jean-François (III): No, no se puede. Eso depende del calor que absorba: si rebota menos, gana... no... pierde energía cinética.

Cécile (III): Precisamente, si estudiamos la energia mecánica.

Jean-François (III): Cuando está abajo, pierde energía cinética.

\section{Cécile (HI): Gana energia potencial.}

Jean-François (III): Por tanto, se calienta, gana energía calorífica.

Al final, aunque han realizado un trabajo experimental idéntico al de Corinne y Ludovic e, incluso, de forma mucho más cuidađosa, Cécile y Jean-François no ban validado su conjetura de proporcionalidad.

\section{CONCLUSIÓN}

Si se considera que el aprendizaje no es solamente una adquisición de saberes sino también la capacidad de dominat esos saberes de forma autónoma, es necesario proponer a los alumnos actividades que les permitan hacer algo más que reproducir los procedimientos (aunque esto siga siendo, evidentemente, útil en un cierto momento deI aprendizaje). Estas actividades deben permitir (y revalorizar) la verificación, pero también la formulación y la validación de conjeturas, en el marco del tiempo escolar y de las actividades de clase. Hemos mostrados cómo la formulación del enunciado desempefra un papel decisivo en el desencadenamiento de estas diversas posibilidades. Los enunciados de tipo contrato (la mayoría de los utilizados actualmente) permiten estar seguros de que el alumno sabe aplicar un procedimiento, pero no permiten más que algunas verificaciones algorítmicas y sobre todo verificaciones de tipo contrato, eficaces para el alumno pero no reconocidas por el físico.

No basta con lanzar a los alumnos a una situación experimental para modificar este comportamiento. Si el enunciado evoca, de lejos o de cerca, una situación prototipo, el alumno no utiliza la situación experimental más que para alcanzar los valores numéricos que faltan y se remite a una situación prototipo respecto a la situación precedente, lo cual permite, sin embargo, verificaciones suplementarias sobre los órdenes de magnitud de un resultado experimental, por ejemplo. Éste es el caso que muestran los enunciados de tipo II. Los alumnos han transformado el enunciado en enunciado contrato, puesto que había una relación con el curso. Han alcanzado valores numéricos utilizando el material experimental suministrado. Sin embargo, hemos constatado que se puede salir del matco prototípico si la experimentación resulta desconcertante. Esta situación experimental desconcertante no debe intervenir demasiado pronto, ya que sino resulta demasiado perturbadora para el alumno, que ya no sabe en absoluto cómo abordar la cuestión. La situación más favorabie parece ser la de una experimentación que no invalide más que parcialmente los resultados adquiridos y que permita al alumno progresar por etapas. Por último, un enunciado totalmente desprovisto de referencias a magnitudes físicas (por tanto, al marco de modelización del sistema) induce inmediatamente a actividades experimentales, seguidas de una actividad especulativa, importantes.

Pero, puesto que el alumno busca primero, lo cual es legítimo, reinvertir las magnitudes introducidas en la enseñanza y puesto que es después de haber explorado todas las posibilidades, lo cual consume mucho tiempo, cuando contempla la posibilidad de introducir una nueva magnitud (lo que un grupo ha llamado aquí rigidez), la duración habitual de una lección no permite ir muy lejos en la validación. Ya que el alumno no obtiene un resultado, tampoco lo puede verificar. Esta situación resulta, al final, muy frustrante para los alumpos.

Llegamos así a la conclusión de que, para desarrollar posibilidades de validación por parte de los alumnos, haría falta permitirles trabajar durante un tiempo mucho más amplio del que se utiliza tradicionalmente. El hecho de proponerles, junto a los enunciados normales, enunciados no contractuales, con posibilidades de experimentación, se nos presenta como un medio interesante para modificar el comportamiento de los alumnos. Pasaríamos así de la investigación de la comprensión del enunciado a una investigación de la comprensión del fenómeno. Nuestra experiencia muestra que ello no exige medios experimentales importantes, sino que basta con interesarse por fenómenos no tratados en los programas. 


\section{NOTAS}

1 Hipótesis es un término corriente para un físico; los enseñantes prefieren conjetura.

2 El coeficiente de restitución se define como la razón de las velocidades relativas normales de dos objetos después de chocar:

$V_{r_{1}}=\mathrm{e} \cdot \mathrm{Vr}_{r_{2}}$

$0<\mathrm{e}<\mathrm{I}$ si $^{2} \mathrm{e}=0$, el choque es blando

si $\mathrm{e}=1$, el choque es perfectamente elástico.

$\mathrm{V}_{\mathrm{r}_{1}}=\mathrm{V}_{1}-\mathrm{V}^{\prime}$

$\mathrm{Vr}_{2}=\mathrm{V}_{2}-\mathrm{V}_{2}^{\prime}$

$V_{1}$ y $V_{2}$, velocidades antes del choque

$V_{1}^{\prime} y_{2} V_{2}$, velocidades después del choque

Aquí, los objetos son la bola y el suelo.
${ }^{3}$ El término modelo se toma aquí en su sentido blando de «herramienta teórica reconociđa como pertinente, en física, para resolver el problema».

${ }^{`}$ Comprensión, igual que explicación, más adelante, se toma en su acepción general (Gréa 1993).

${ }^{5}$ Nosotros atribuimos la primera interpretación a la presencia en la sala de un utillaje del tipo plano inclinado que los alumnos ya habían utilizado para el estudio de los rozamientos y que, implícitamente, han cré́do que debían utilizar.

* Este artículo ha sido traducido del francés por Jaime Tortella.

\section{REFERENCIAS BIBLIOGRÁFICAS}

BROUSSEAU, G., 1986. Fondements et méthodes de ladidactique des mathématiques, Recherche en didactique des mathematique, Vol. 7(2).

BRUHAT, G., 1948. Cours de Physique générale, Mécanique physique, Masson éditeurs.

CHI, M.T.H., FELTOVITCH, P.J, y GLASER R., 1981. Categorisation and representation of physics problems by experts and novices, Cognitive Sciences, 5(2), pp. 121-152.

DUMAS-CARRÉ A., CAILLOT M., MARTÍNEZ TORREGROSA, J. y GIL D., 1989. Deux approches pour modiffer les activités de résolution de problèmes en physique dans l'enseignement secondaire: une tentative de synthèse, Aster, 8, pp. 135-160.

DUMAS-CARRÉ, A., 1987. La résolution de problème en physique au lycée. Thèse d'état, París, 7 de junto, 1987.

GIL, D. y MARTÍNEZ TORREGROSA J., 1987. La résolution de problème comme instrument de changement conceptuel et méthodologique, Petit $X, 14-15$, pp. 25-38.

GRÉA, J., 1993. Modèle, niveaux de formulation et explication, IIİme Séminaite National de Didactique des Sciences Physique, Toulouse, 1993.
HOC, J.M., 1987. Psyckologie cogritive de Ia planification, Presse Universitaire de Grenoble.

KHANTINE-LANGLOIS, F., 1988. Problèmes de physique en 1ère $S$ : étude de quelques critères de vérification, mémoire de DEA Université Lyon 1.

LARKIN, I.H., MCDERMOTT J., SIMON, D.P. y SIMON H.A., 1980. Expert and novice performances in solving physics problems, Science, 108, pp. 1335-1342.

LARKIN, J.H., 1983. The role of problem representation in Physics, en Gentner, D., Stevens, A. (eds.), Mental Models Hillside Laurence Erbaum Associated.

Programa BO, núm. especial, 322 , abril de 1982.

Physique 1 S E 1982, Hatier.

Physique 1 S E 1982, Nathan.

TIBERGHIEN, A. y LANGLOIS, F., 1991. Modelling in physics and problem solving. The case of mechanical energy at Iycenm level, en Teaching about Reference Frames: from Copenicus to Einstein, Tonan: Nicholas Copernicus University Press. 


\section{Problema Ia: El automóvil (según Hatier Physique I S E} 1982)

Un automóvil cuya masa es de $1200 \mathrm{~kg}$, circula por una carretera rectilinea. Se supone que la fuerza $\mathrm{F}$ que se opone a su desplazamiento no varía más que con la velocidad V. La figura 1 presenta el gráfico de la función $\mathrm{V} \rightarrow \mathrm{F}$.

1) ¿Cuál es la fuerza que se opone al desplazamiento del automóvil cuando $\mathrm{V}=54 \mathrm{~km} / \mathrm{h}$ ?

2) La carretera es horizontal:

a) ¿Cuáles son las fuerzas que actúan sobre al automóvil?

b) Calcular los trabajos de esas fuerzas para un desplazamiento de $1 \mathrm{~km}$ a la velocidad de $54 \mathrm{~km} / \mathrm{h}$.

c) Calcular la potencia de la fuerza motriz del automóvil circulando a $54 \mathrm{~km} / \mathrm{h}$.

3) La carretera es una pendiente del 5\% (para un recorrido de $100 \mathrm{~m}$, el desnivel es de $5 \mathrm{~m}$ ). Responder a las mismas preguntas $a, b$ y $c$ cuando el automóvil circula subiendo a lo largo de $1 \mathrm{~km}$.

\section{Problema Ib: El péndulo (Nathan Physique 1 S E edición} 1982)

Una varilla $S$ de centro de inercia $G$, puede girar alrededor de un eje horizontal $D$ que la atraviesa perpendicularmente en $O$ (Fig. 2). Sea d(O,G) =a = 0,25 m; la posición de la varilla viene marcada por el ángttlo que forma $O G$ con la vertical $O y$. Se da la representación gráfica de la energía potencial del sistema (varitla - tierra) en función dei ángulo (Fig. 3). El momento de inercia de la varilla tiene la expresión $\mathrm{y}=\frac{1}{3} \mathrm{~m} \mathrm{l}^{2}$, siendo $\mathrm{m}$ la masa de la varilla y $l$ su longitud, $1=2 a, m=480 \mathrm{~g}$.

1) Teniendo en cuenta la curva 2 , decir cuál es la posición adoptada por convenio para el nivel de la energía potencial nula.

2) Estando la varilla $S$ en rotación alrededor del eje D, habrá dos valores posibles de la energía mecánica total, los de las rectas 1 y 2 .

Primer caso: La energía mecánica total viene representada por la recta 1.

a) Demostrar que la trayectoria es un arco de círculo, del que se pide la medida en grados.

b) ¿Cuát es el valor de la velocidad de $G$ al pasar por la posición de equílibrio?

c) Determinar, con la ayuda del gráfico, las posiciones de $\mathrm{S}$ cuando su energía vale 0'4 julios.

Segundo caso: La energía mecánica total viene representada por la recta 2 .

a) ¿Cuál es la trayectoria de $G$ ?

b) Calcular los valores V1 y V2 de las velocidades máxima y mínima de $G$. Representar los vectores de velocidad V1 y V2 mediante un esquema que exprese la posición de $\mathrm{S}$ en el instante correspondiente.
Figura 1

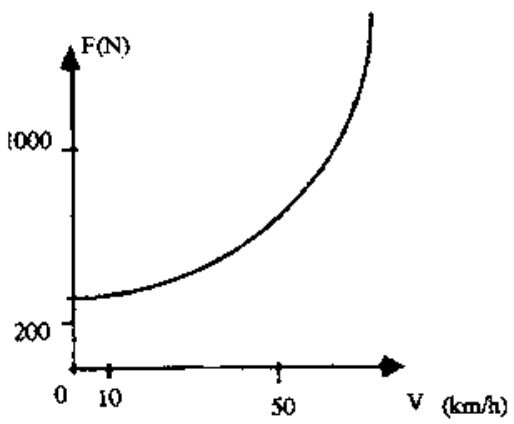

Figura 2

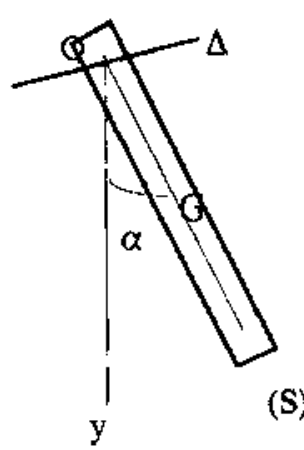

Figura 3

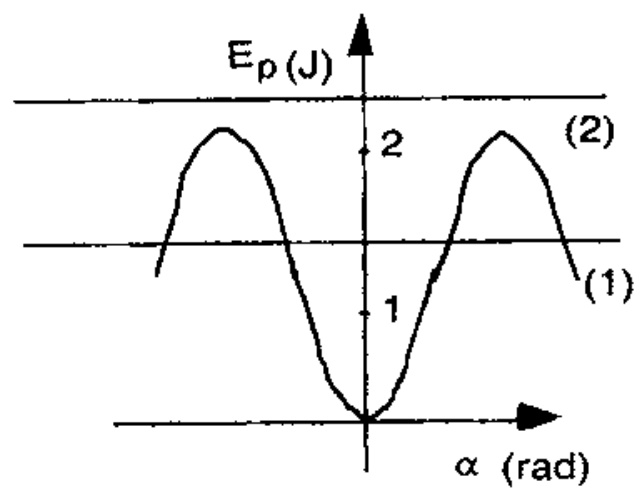




\section{Problema II}

Se pretende comparar una de las propiedades que permiten distinguir las sustancias constituyentes de las bolas. Para ello, se estudian sus comportamientos respectivos mediante sus interacciones con el suelo. Se interpretará el fenómeno en términos de energía.

\section{Problema III}

Disponemos de diversas bolas. ¿Cómo podréis caracterizar su capacidad de rebotar estudiando el primer rebote? Disponéis de otras bolas. ¿Qué predeciréts, siempre en el primer rebote, para esas bolas hechas de otra sustancia? 
